\title{
RESEARCH
}

\section{Predicting the relationships between gut microbiota and mental disorders with knowledge graphs}

Ting Liu' ${ }^{1,2}$, Xueli Pan ${ }^{1}$, Xu Wang ${ }^{1}$, K. Anton Feenstra ${ }^{2}$, Jaap Heringa ${ }^{2}$ and Zhisheng Huang ${ }^{1,3^{*}}$

\begin{abstract}
Gut microbiota produce and modulate the production of neurotransmitters which have been implicated in mental disorders. Neurotransmitters may act as 'matchmaker' between gut microbiota imbalance and mental disorders. Most of the relevant research effort goes into the relationship between gut microbiota and neurotransmitters and the other between neurotransmitters and mental disorders, while few studies collect and analyze the dispersed research results in systematic ways. We therefore gather the dispersed results that in the existing studies into a structured knowledge base for identifying and predicting the potential relationships between gut microbiota and mental disorders. In this study, we propose to construct a gut microbiota knowledge graph for mental disorder, which named as MiKG4MD. It is extendable by linking to future ontologies by just adding new relationships between existing information and new entities. This extendibility is emphasized for the integration with existing popular ontologies/terminologies, e.g. UMLS, MeSH, and KEGG. We demonstrate the performance of MiKG4MD with three SPARQL query test cases. Results show that the MiKG4MD knowledge graph is an effective method to predict the relationships between gut microbiota and mental disorders.
\end{abstract}

Keywords: Knowledge graph, Mental disorders, Neurotransmitters, Microbiota-gut-brain axis, Biomedical ontology, Gut microbiota

\section{Introduction}

The microbiota-gut-brain axis used to describe the complex networks and relationships between gut microbiota and the host, which reflects the inextricable association between gut microbiota and the mental health of the host [1]. A growing body of evidence points toward that gut microbiota play a role in the development of mental disorders [2]. The composition and diversity of gut microbiota in depressed patients

\footnotetext{
*Correspondence: z.huang@vu.nl

${ }^{3}$ Brain Protection Innovation Center, Capital Medical University, Beijing, China

Full list of author information is available at the end of the article
}

significantly differ from those in healthy controls [3]. Gut microbiota have been implicated in many different mental disorders, e.g. eating disorders [4] and sleeping disorders [5], in humans. The underlying theory is that gut microbiota influence the mental health of the host by regulating the level of neurotransmitters [6]. On the one hand, gut microbiota produce or modulate the production of neurotransmitters [7]. Lactobacillus plantarum, a lactic acid-producing bacterium, increased both serotonin and dopamine levels in germ-free mice [8]. The family of Bacillus and Escherichia generate dopamine and/or norepinephrine [9], while GABA produced by the certain species of Lactobacillus [6]. Gut microbiota promote the synthesis of histamine [10] and acetylcholine [11] in vivo. On the other hand, these neurotransmitters have been most studied concerning

(c) The Author(s) 2020. This article is licensed under a Creative Commons Attribution 4.0 International License, which permits use, sharing, adaptation, distribution and reproduction in any medium or format, as long as you give appropriate credit to the original author(s) and the source, provide a link to the Creative Commons licence, and indicate if changes were made. The images or other third party material in this article are included in the article's Creative Commons licence, unless indicated otherwise in a credit line to the material. If material is not included in the article's Creative Commons licence and your intended use is not permitted by statutory regulation or exceeds the permitted use, you will need to obtain permission directly from the copyright holder. To view a copy of this licence, visit http://creativecommons.org/licenses/by/4.0/. 
mental disorders [12]. Interruptions of serotonin and norepinephrine movement lead to depression and anxiety disorders [13]. Dopamine is another neurotransmitter linked to mental disorders, such as schizophrenia and autism spectrum disorder $[14,15]$. Sleep and eating disorders are believed to be the results of interrupted passages of dopamine, norepinephrine, or GABA [16, 17]. Histamine and acetylcholine have well-established roles in the regulation of cognition disorders [18]. Additional mental disorders, such as personality disorders and sex behavior disorder, have been proven to be caused by the interrupted transfer of neurotransmitter messages $[19,20]$. The imbalance of neurotransmitters is one reason for the distress or impairment of personal mental health. The associations between neurotransmitters and mental disorders, and the production of neurotransmitters by various members of the gut microbial community, suggest that gut microbiota may influence the mental health of the host by regulating the level of neurotransmitters.

We hypothesis that neurotransmitters act as 'matchmaker' between gut microbiota imbalance and mental disorders. Collecting and analyzing the relationships between gut microbiota and neurotransmitters, as well as neurotransmitters and mental disorders, benefit for identifying and predicting the implicit relationships between gut microbiota and mental disorders. We therefore aim to gather the disparate results in existing studies into a structured knowledge base. Knowledge graphs help to combine isolated results, giving an overview of the knowledge in an area [21]. This technique is able to connect and represent semi-structured or unstructured data in a systematic way [21, 22], and supports semantic searching, question answering, and visual decision supporting [23-25]. Currently, knowledge graphs have been widely used in medicine, such as comorbidity analysis [26], drug discovery [27], healthcare services [28], medical health status classification [29], and predicting relationships between microbes and human diseases [30].

In this study, we proposed a novel knowledge graph which we named as MiKG4MD to identify and predict the relationships between gut microbiota and mental disorders. Because of the separation of the knowledge base and the library of algorithm program, MiKG4MD is extendable by linking to future ontologies by just adding new relationships between existing information and new entities. We extended the knowledge base by integrating with existing popular biomedical ontologies, e.g. Unified Medical Language System (UMLS), Kyoto Encyclopedia of Genes and Genomes (KEGG), and Medical Subject Headings (MeSH). We demonstrate the performance of MiKG4MD with three SPARQL test cases. Results show that the MiKG4MD knowledge graph is an effective method to predict the relationship between gut microbiota and mental disorders. Constructing such a knowledge graph that gathers existing knowledge resources not only enables users to achieve semantic queries and question answering but may also be supporting medical researchers to make better decisions to implement novel therapies for various mental diseases.

\section{Methodology \\ Data sources collection}

Currently, more than 200 neurotransmitters have been identified. We here only take account of six major neurotransmitters, i.e. serotonin, dopamine, GABA, norepinephrine, histamine, and acetylcholine, which are often implicated in the pathogenesis of mental disorders. Google Scholar and PubMed literature search combined the terms 'gut microbiota,' 'gut flora,' 'intestinal bacteria, 'neurotransmitter', 'serotonin', 'dopamine', 'norepinephrine', 'GABA', 'histamine', and 'acetylcholine' to identify studies that investigated relationships between gut microbiota and neurotransmitters. With no limitation of study design, all relevant articles were carefully reviewed by three researchers. Finally, thirty-five articles on the regulation between gut microbiota and neurotransmitters were identified for further extraction of entities and relations. The evidence level of these studies was ranked from A to E according to their strength of the randomized controlled trial design as we presented in another paper [6]. References of the relationship between neurotransmitters and mental disorders were identified through a literature search on PubMed and Google Scholar with keywords: serotonin, dopamine, norepinephrine, GABA, histamine, acetylcholine, anxiety disorders, depressive disorder, sleep disorders, eating disorders, sex behavior disorder, personality disorder, bipolar disorder, autistic disorder, cognition disorders, and learning disorders. Due to the huge amount of references for each relationship, we limit the number of references to no more than five which we selected randomly.

\section{Data extraction and structure}

To construct a knowledge graph, the most important process is to extract the entities and relations from available data sources. Many text mining tools that allow users to extract knowledge from free text, but they all require a large number of the training datasets. Even so, they often fail when encounter new terms. It was therefore decided to manually obtain highly accurate annotation of entities and semantic relations from the free text, which done by the authors. In this study, we have several classes of annotations, divided among 'entities' (neurotransmitter, mental disorder, gut microbiota, and KEGG 
pathway), and 'relations' (reference, statement, and relationship). The six neurotransmitters make up the class 'Neurotransmitter'. Class 'Mental disorders' contains ten common mental disorders, including anxiety disorders, depressive disorder, sleep disorders, eating disorders, sex behavior disorder, personality disorder, bipolar disorder, autistic disorder, cognition disorders, and learning disorders. The forty-five entities of gut microbiota that extracted from studies constitute the class 'Gut microbiota.' Class 'KEGG pathway' includes fifty-six pathways of the six neurotransmitters. Class 'Statement' is used to describe the semantic relational properties between gut microbiota and neurotransmitters, while 'Relationship' is used to describe the relations between neurotransmitters and mental disorders. We use the Terse RDF Triple Language (Turtle) format to structure the extracted entities and concepts with relations.

\section{Knowledge base enrichment}

We enriched the semantic database by integrating with existing biomedical ontologies/terminologies, i.e. UMLS, $\mathrm{MeSH}$, and KEGG databases. The UMLS is a repository of biomedical vocabularies and covers well known medical terminologies [31]. Each Metathesaurus concept in UMLS has a single Concept Unique Identifier (CUI) which links the concept data across files. The $\mathrm{MeSH}$ is a comprehensive controlled vocabulary thesaurus, used for indexing journal articles and books in the life sciences [32]. Each entry is identified by the MeSH Unique ID that accompanied by a definition, links to related descriptors, a list of entry terms, and role relationships in $\mathrm{MeSH}$ categories. In this paper, we link the concepts of gut microbiota, neurotransmitters, and mental disorders to UMLS and $\mathrm{MeSH}$ by matching the CUI and $\mathrm{MeSH}$ ID. The KEGG compound database is a collection of small molecules, biopolymers, and other chemical substances that are relevant to biological systems. Each entry is identified by the $C$ number that contains its chemical structure and associated relationships, along with various links to other databases. KEGG pathway database is a collection of pathway maps on the molecular interaction, cellular processes, organismal systems, and human diseases [33]. Each entry is identified by the map number. Here we link the neurotransmitters with KEGG databases for further research purposes.

\section{Knowledge graph visualization}

A knowledge graph consists of a set of concepts (classes), a set of attributes (data type properties), relationships (object properties), and constraints to abstractly represent a specific event [34]. Visualization of MiKG4MD provides a clear overview of the hierarchy and connections within the knowledge base, which is an important step in the process of knowledge graph construction. Figure 1 depicts the visualization of our knowledge graph. A knowledge base can be conceptually represented as a collection of terminologies (TBox) and assertions (ABox) [35]. TBox is used to describe a domain of interest by defining classes and properties as a domain vocabulary as shown in blue part in Fig. 1. ABox is TBox-compliant statements about individuals belonging to these sets, as shown in the pink part in Fig. 1. Nodes in rounded rectangle labeled with KEGG, Literal, and MeSH ID link internal classes with external concepts in other databases. Therefore, the constructed knowledge graph can integrate the wealth of information available from biomedical databases for semantic enrichment.

\section{Case study}

We design three test cases to demonstrate the identification and prediction performance of the knowledge graph by using the SPARQL query. Users can perform their own queries by changing parameters as desired in templates that we designed. The MiKG4MD knowledge base, SPARQL query codes, and results of three cases are available at GitHub. ${ }^{1}$ Generally a SPARQL query contains three components. The PREFIX at top defines the list of ontologies that used in a query. The SELECT DISTINCT statement is used to return only distinct values. The WHERE clause is used to extract only those records that fulfill a specified condition. We use several abbreviations in the following query codes and results. 'Ref' is an abbreviation for reference. 'Level' refers to the evidence level the reference. 'PMID' is the unique identifier number of a reference in PubMed. Other abbreviations: GM, gut microbiota; NTM, neurotransmitter; MD, mental disorder. 




Table 1 The query result of test case 1

\begin{tabular}{|c|c|c|c|c|c|c|c|}
\hline & GM & NTM & Ref & PMID & Level & MD1 & MD2 \\
\hline 1 & Bifidobacterium dentium & GABA & DeVadder 2018 & 29866843 & B & Anxiety disorders & Depressive disorder \\
\hline 2 & Bifidobacterium dentium & GABA & Pokusaeve 2017 & 27458085 & B & Anxiety disorders & Depressive disorder \\
\hline 3 & Bifidobacterium dentium & GABA & Barrett 2012 & 22612585 & C & Anxiety disorders & Depressive disorder \\
\hline
\end{tabular}


Table 2 The query result of test case 2

\begin{tabular}{|c|c|c|c|c|c|c|c|}
\hline & GM & NTM & Modulate & Level & Ref & PMID & MD \\
\hline 1 & Lactobacillus plantarum & Acetylcholine & Increase & C & Stanasze 1997 & 907345 & Depressive disorder \\
\hline 2 & Lactobacillus plantarum & Acetylcholine & Increase & C & Stanasze 1997 & 907345 & Sleep disorders \\
\hline 3 & Lactobacillus plantarum & Acetylcholine & Increase & C & Stanasze 1997 & 907345 & Sex behavior disorder \\
\hline 4 & Lactobacillus plantarum & Acetylcholine & Increase & C & Stanasze 1997 & 907345 & Bipolar disorder \\
\hline 5 & Lactobacillus plantarum & Acetylcholine & Increase & C & Stanasze 1997 & 907345 & Cognition disorders \\
\hline 6 & Lactobacillus plantarum & Acetylcholine & Increase & C & Stanasze 1997 & 907345 & Autistic disorder \\
\hline
\end{tabular}

Lactobacillus plantarum associated with mental disorders by increasing the level of acetylcholine

GM gut microbiota, NTM neurotransmitter, MD mental disorder

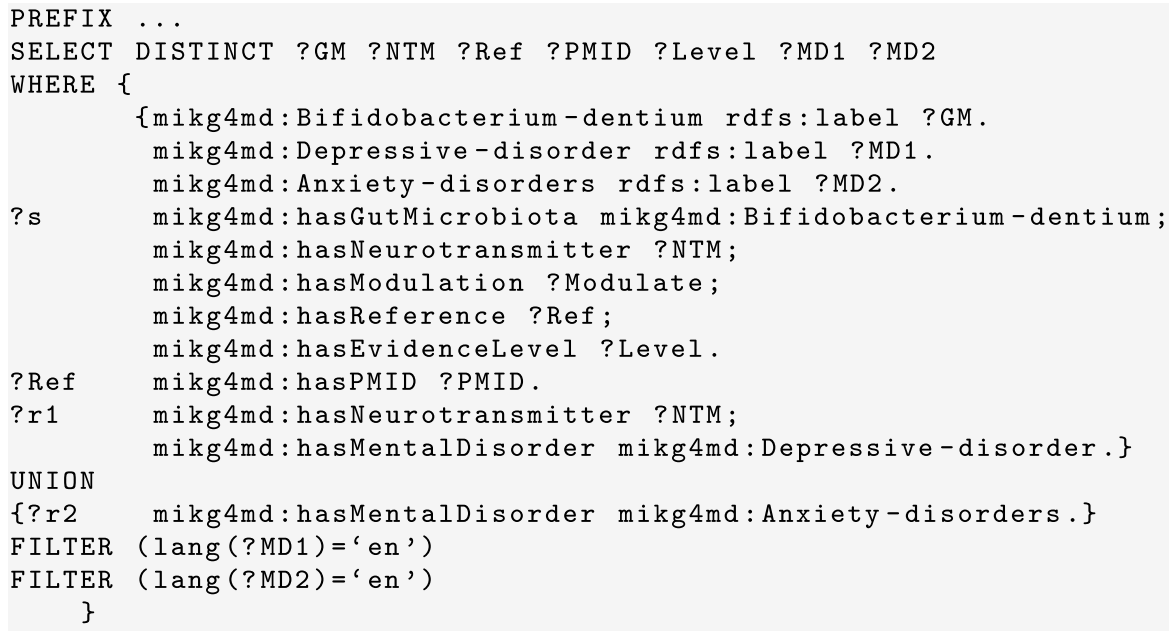

\section{Test case 1: gut microbiota based query}

We consider a situation that a person encounters anxiety disorder and depressive disorder. We are interested in if Bifidobacterium dentium, an important gut bacteria of humans, may be related to anxiety disorder and depressive disorder by regulating the level of some neurotransmitters. The SPARQL query code is presented at Listing 1 . In this query, Bifidobacterium dentium is a given condition to semantic search its related values. Therefore, we define Bifidobacterium dentium as the gut microbiota to query its related variables, such as neurotransmitters, reference, and mental disorder. We expect to show the reference with its PMID number, we therefore asked it after the statement. A UNION is used to combine results of anxiety disorder and depressive disorder. We apply two FILTER conditions to select labels of mental disorder in English, as shown in Listing 1. The obtained results indicate that GABA is the only neurotransmitter that modulated by Bifidobacterium dentium, which is supported by three references: two level $\mathrm{B}$ and one level $\mathrm{C}$ as shown in Table 1 . In addition, Bifidobacterium dentium may relate to anxiety disorder and depressive disorder by regulating the level of GABA. 
Table 3 The query result of test case 3

\begin{tabular}{|c|c|c|c|c|c|c|c|}
\hline & GM & NTM1 & NTM2 & Level & Ref & PMID & MD \\
\hline 1 & Clostridium & Dopamine & Norepinephrine & B & Asano 2012 & 23064760 & Bipolar disorder \\
\hline 2 & Escherichia coli & Dopamine & Norepinephrine & C & Shishov 2009 & 19845286 & Bipolar disorder \\
\hline 3 & Bacillus mycoides & Dopamine & Norepinephrine & C & Tsavkelova 2000 & 10935181 & Bipolar disorder \\
\hline 4 & Bacillus subtilis & Dopamine & Norepinephrine & C & Tsavkelova 2000 & 10935181 & Bipolar disorder \\
\hline 5 & Escherichia coli & Dopamine & Norepinephrine & C & Tsavkelova 2000 & 10935181 & Bipolar disorder \\
\hline 6 & Proteus vulgaris & Dopamine & Norepinephrine & C & Tsavkelova 2000 & 10935181 & Bipolar disorder \\
\hline 7 & Serratia marcescens & Dopamine & Norepinephrine & C & Tsavkelova 2000 & 10935181 & Bipolar disorder \\
\hline
\end{tabular}

Some species of gut microbiota associated with bipolar disorder by affecting dopamine and norepinephrine levels

GM gut microbiota, NTM neurotransmitter, MD mental disorder

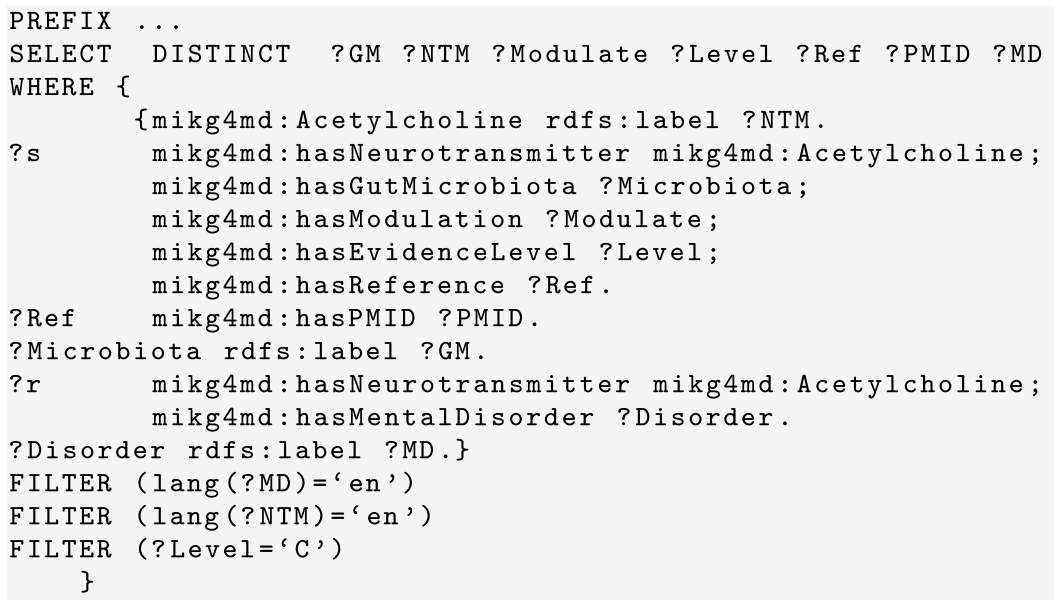

\section{Test case 2: neurotransmitter based query}

Acetylcholine serves as a primarily excitatory neurotransmitter in the central nervous system [6]. It plays a role in arousal, memory, and learning [36]. It is well known that gut microbiota generate acetylcholine. We therefore aim to investigate which gut microbiota species may be related to mental disorders by modulating acetylcholine levels. The query code we designed is shown in the Listing 2 . We define the acetylcholine as the neurotransmitter to query its related variables, like gut microbiota, reference, PMID, and mental disorder. We show the labels of neurotransmitters and mental disorders in English by applying FILTER conditions after the WHERE clause. Besides this, we use FILTER to obtain only level $\mathrm{C}$ evidence in this case (Listing 2). In MiKG4MD, Lactobacillus plantarum is the only species of gut microbiota that modulate the acetylcholine levels, as shown in Table 2. This fact comes from one reference with level $\mathrm{C}$ evidence, and the PMID of the reference is 907345. The result shows that Lactobacillus plantarum has effects on six different mental disorders by regulating the level of acetylcholine. The six mental disorders are depressive disorder, sleep disorders, sex behavior disorder, bipolar disorder, cognition disorders, and autistic disorder. 


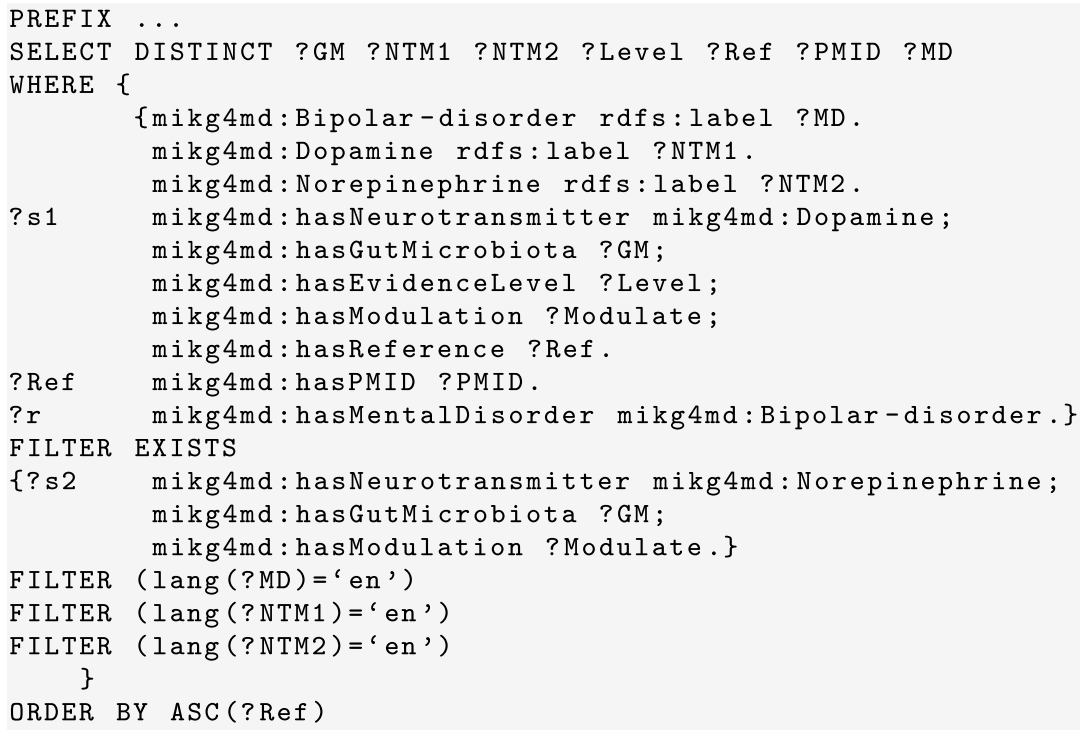

\section{Test case 3: mental disorder based query}

Bipolar disorder is a serious mental disorder in which a person experiences extreme variances in mood, energy, thinking, and behavior [37]. The neurotransmitters that are implicated in bipolar disorder include dopamine, norepinephrine, serotonin, GABA, and acetylcholine [38]. We are interested in which species of gut microbiota may relate to bipolar disorder by regulating the neurotransmitter levels. For this specific condition, we here only take dopamine and norepinephrine into consideration, but not mean the others not important. We dropped the SPARQL query code at Listing 3. In this query, dopamine, norepinephrine, and bipolar disorder are the given conditions. We design the WHERE clause to find the variables according to the given conditions. At this stage, we use FILTER EXISTS to achieve the intersection result of serotonin and dopamine. FILTER conditions used to select the labels of neurotransmitters and mental disorders in English (Listing 3). As shown in Table 3, one level B evidence proved that species of Clostridium have effects on the content of dopamine and norepinephrine. In addition, species Escherichia coli, Bacillus mycoides, Bacillus subtilis, Proteus vulgaris, and Serratia marcescens modulate the levels of those two neurotransmitters. These facts come from two references with level $\mathrm{C}$ evidence. These species of gut microbiota strongly associated with bipolar disorder by altering dopamine and norepinephrine levels.

\section{Discussion and conclusion}

Gut microbiota influence mental health by producing neurotransmitters directly or regulating the relevant metabolism pathways of neurotransmitters [39]. To predict the relationships between gut microbiota and mental disorders with neurotransmitters as the linking element, we first constructed a knowledge base by integrating the disparate knowledge from existing studies in a semantic way. MiKG4MD, the novel knowledge graph we proposed, enables us to identify and predict the potential connections by semantic querying and reasoning. In the process of constructing such a domain knowledge graph, relationships can be divided into explicit relationships and implicit relationships.

The support of a knowledge graph for practical applications much depends on the construction of implicit relationships. Therefore, the potential for discovering implicit relationships is crucial for a domain knowledge graph. We designed three test cases to demonstrate the potential identification and prediction performance of the knowledge graph by using the SPARQL query. The results show that gut microbiota are related to mental disorders with neurotransmitters as link. From these cases, we have learned that our knowledge graph has the potential for discovering implicit relationships. Taken together, MiKG4MD is an effective knowledge graph to identify, explore and predict the relationships between gut microbiota and mental disorders. 


\section{Limitations and outlook}

The main limitation of MiKG4MD is its limited coverage. The number of entities and relations of gut microbiota, neurotransmitters, and mental disorders are by necessity limited, which results in the query result being correct but incomplete, i.e. not covering the whole knowledge domain. As we know, gut microbiome consists of many trillions of microorganisms, including at least a thousand different species of known bacteria [40], while over 200 neurotransmitters have been identified so far [41]. Besides, there are over 300 different mental disorders listed in the DSM-5 (Diagnostic and Statistical Manual of Mental Disorders) [42]. To be sure, gut microbiota do have an impact on mental health by regulating neurotransmitters, but that is not as simple as one bacterium-one neurotransmitter. Therefore, our future work will emphasize on automated enrichment of the knowledge graph. For completely understanding the role of gut microbiota and neurotransmitters in mental disorders with a knowledge graph, like MiKG4MD, more entities and relations should be included in its knowledge base. Besides this, in the current study, we extracted entities and relations manually to ensures accuracy of the data. Manual processing, however, face huge challenges when dealing with large and wide datasets. We expect the knowledge graph would not have to be updated manually to have new information placed within it. Let the knowledge graph update itself when it collects information about entities, their properties and attributes, and relationships involving them. To realize the automation of knowledge extraction and regular updating of the database, other advanced technology (including deep learning [43-45], neuroevolution [46, 47], and evolutionary algorithms [48-50], will be employed in our future works.

\begin{abstract}
Author contributions
T.L. and Z.H. designed the project. T.L., X.P. and X.W. performed the data sources collection, data extraction and structure. T.L., X.P. and Z.H. designed and performed the test cases. Z.H. K.A.F. and J.H. supervised and directed the work continuously. T.L. wrote the manuscript with contributions from all coauthors. All authors provided critical feedback and helped shape the research, analysis and manuscript.
\end{abstract}

\section{Funding}

This work was partially supported by the China Scholarship Council.

\section{Data availability}

The data and code that support the findings of this study are openly available at GitHub (https://github.com/tingcosmos/MiKG4MD.git).

\section{Compliance with ethical standards}

\section{Conflict of interest}

The authors declare that they have no competing interests.

\section{Author details}

${ }^{1}$ Knowledge Representation and Reasoning (KR\&R) Group, Vrije Universiteit Amsterdam, Amsterdam, The Netherlands. ${ }^{2}$ Center for Integrative Bioinformatics VU (IBIVU), Vrije Universiteit Amsterdam, Amsterdam, The Netherlands.

${ }^{3}$ Brain Protection Innovation Center, Capital Medical University, Beijing, China.

Received: 24 August 2020 Accepted: 30 September 2020

Published online: 24 November 2020

References

1. Rieder R, Wisniewski PJ, Alderman BL, Campbell SC. Microbes and mental health: a review. Brain Behav Immun. 2017;66:9-17.

2. Järbrink-Sehgal $\mathrm{E}$, Andreasson A. The gut microbiota and mental health in adults. Curr Opin Neurobiol. 2020;62:102-14.

3. Zheng P, Zeng B, Zhou C, Liu M, Fang Z, Xu X, Zeng L, Chen J, Fan S, Du $X$, et al. Gut microbiome remodeling induces depressive-like behaviors through a pathway mediated by the host's metabolism. Mol Psychiatry. 2016;21(6):786

4. Kleiman SC, Watson HJ, Bulik-Sullivan EC, Huh EY, Tarantino LM, Bulik CM, Carroll IM. The intestinal microbiota in acute anorexia nervosa and during renourishment: relationship to depression, anxiety, and eating disorder psychopathology. Psychosom Med. 2015;77(9):969.

5. Smith RP, Easson C, Lyle SM, Kapoor R, Donnelly CP, Davidson EJ, Parikh E, Lopez JV, Tartar JL. Gut microbiome diversity is associated with sleep physiology in humans. PLoS ONE. 2019;14(10):e0222394.

6. Liu T, Feenstra KA, Heringa J, Huang Z. Influence of gut microbiota on mental health via neurotransmitters: a review. J Artif Intell Med Sci. 2020;1:1-14.

7. Liu T, Huang Z. Evidence-based analysis of neurotransmitter modulation by gut microbiota. In: International Conference on Health Information Science, pp. 238-249. Springer (2019).

8. Liu W-H, Chuang H-L, Huang Y-T, Chien-Chen W, Chou G-T, Wang S, Tsai Y-C. Alteration of behavior and monoamine levels attributable to lactobacillus plantarum ps128 in germ-free mice. Behav Brain Res. 2016;298:202-9.

9. Lyte M. Probiotics function mechanistically as delivery vehicles for neuroactive compounds: microbial endocrinology in the design and use of probiotics. BioEssays. 2011;33(8):574-81.

10. Diaz M, del Rio B, Ladero V, Redruello B, Fernandez M, Martin MC, Alvarez MA. Isolation and typification of histamine-producing lactobacillus vaginalis strains from cheese. Int J Food Microbiol. 2015;215:117-23.

11. Valles-Colomer M, Falony G, Darzi Y, Tigchelaar EF, Wang J, Tito RY, Schiweck C, Kurilshikov A, Joossens M, Wijmenga C, Claes S. The neuroactive potential of the human gut microbiota in quality of life and depression. Nat Microbiol. 2019;4:623-32.

12. Brown RP, Mann JJ. A clinical perspective on the role of neurotransmitters in mental disorders. Psychiatric Serv. 1985;36(2):141-50.

13. Montoya A, Bruins R, Katzman MA, Blier P. The noradrenergic paradox: implications in the management of depression and anxiety. Neuropsychiatr Dis Treatment. 2016;12:541.

14. Brisch R, Saniotis A, Wolf R, Bielau H, Bernstein H-G, Steiner J, Bogerts B, Braun K, Jankowski Z, Kumaratilake J, et al. The role of dopamine in schizophrenia from a neurobiological and evolutionary perspective: old fashioned, but still in vogue. Front Psychiatry. 2014;5:47.

15. Pavăl D. A dopamine hypothesis of autism spectrum disorder. Dev Neurosci. 2017;39(5):355-60.

16. Brown RE, Basheer R, McKenna JT, Strecker RE, McCarley RW. Control of sleep and wakefulness. Physiol Rev. 2012;92(3):1087-187.

17. Avena NM, Bocarsly ME. Dysregulation of brain reward systems in eating disorders: neurochemical information from animal models of binge eating, bulimia nervosa, and anorexia nervosa. Neuropharmacology. 2012;63(1):87-96.

18. Blandina P, Efoudebe M, Cenni G, Mannaioni P, Passani MB. Acetylcholine, histamine, and cognition: two sides of the same coin. Learning Memory. 2004;11(1):1-8.

19. Gurvits IG, Koenigsberg HW, Siever LJ. Neurotransmitter dysfunction in patients with borderline personality disorder. Psychiatr Clin N Am. 2000;23(1):27-40 
20. Frank JE, Mistretta P, Will J. Diagnosis and treatment of female sexual dysfunction. Am Fam Phys. 2008;77(5):635-42.

21. Cudré-Mauroux P. Leveraging knowledge graphs for big data integration: the xi pipeline. Semantic Web. 2020;11(1):13-7.

22. Huang Z, Yang J, van Harmelen F, Hu Q. Constructing knowledge graphs of depression. In International Conference on Health Information Science, pp. 149-161. Springer; 2017.

23. Zheng W, Zou L, Peng W, Yan X, Song S, Zhao D. Semantic sparql similarity search over rdf knowledge graphs. Proc VLDB Endow. 2016:9(11):840-51.

24. Moschitti A, Tymoshenko K, Alexopoulos P, Walker A, Nicosia M, Vetere G, Faraotti A, Monti M, Pan JZ, Wu H, Zhao Y. Question answering and knowledge graphs. In: Pan JZ, Vetere G, Gomez-Perez JM, Wu H, editors. Exploiting linked data and knowledge graphs in large organisations. Cham: Springer; 2017. p. 181-212.

25. Elnagar S, Weistroffer HR. Introducing knowledge graphs to decision support systems design. In: Wrycza S, Maślankowski J, editors. Information systems: research, development, applications, education. Cham: Springer; 2019. p. 3-11.

26. Biswas S, Mitra P, Rao KS. Relation prediction of co-morbid diseases using knowledge graph completion. IEEE/ACM Trans Comput Biol Bioinform. 2019;99:1.

27. Sang S, Yang Z, Liu X, Wang L, Lin H, Wang J, Dumontier M. Gredel: a knowledge graph embedding based method for drug discovery from biomedical literatures. IEEE Access. 2018;7:8404-15.

28. Shi L, Li S, Yang X, Qi J, Pan G, Zhou B. Semantic health knowledge graph: semantic integration of heterogeneous medical knowledge and services. BioMed Res Int. 2017:2017:2858423.

29. Pham T, Tao X, Zhang J, Yong J. Constructing a knowledge-based heterogeneous information graph for medical health status classification. Health Inform Sci Syst. 2020;8(1):1-14.

30. Janssens Y, Nielandt J, Bronselaer A, Debunne N, Verbeke F, Wynendaele E, Van Immerseel F, Vandewynckel Y-P, De Tré G, De Spiegeleer B. Disbiome database: linking the microbiome to disease. BMC Microbiol. 2018;18(1):50

31. Olivier B. The unified medical language system (umls): integrating biomedical terminology. Nucleic Acids Res. 2004;32:D267-70.

32. Coletti MH, Bleich HL. Medical subject headings used to search the biomedical literature. J Am Med Inform Assoc. 2001;8(4):317-23.

33. Kanehisa M, Goto S. Kegg: kyoto encyclopedia of genes and genomes. Nucleic Acids Res. 2000;28(1):27-30.

34. Achich N, Bouaziz B, Algergawy A, Gargouri F. Ontology visualization: an overview. In International Conference on Intelligent Systems Design and Applications, pp. 880-891. Springer; 2017.

35. Brachman RJ, Levesque HJ, Fikes R. Krypton. Integrating terminology and assertion. AAAl. 1983;83:31-5.
36. Picciotto MR, Higley MJ, Mineur YS. Acetylcholine as a neuromodulator: cholinergic signaling shapes nervous system function and behavior. Neuron. 2012;76(1):116-29.

37. Kupka RW, Altshuler LL, Nolen WA, Suppes T, Luckenbaugh DA, Leverich GS, Frye MA, Keck PE Jr, McElroy SL, Grunze H, Post RM. Three times more days depressed than manic or hypomanic in both bipolar i and bipolar ii disorder 1. Bipolar Disorders. 2007;9(5):531-5.

38. Shi J, Badner JA, Hattori E, Potash JB, Willour VL, McMahon FJ, Gershon ES, Liu C. Neurotransmission and bipolar disorder: a systematic family-based association study. Am J Med Genet Part B. 2008;147(7):1270-7.

39. Strandwitz P. Neurotransmitter modulation by the gut microbiota. Brain Res. 2018;1693:128-33.

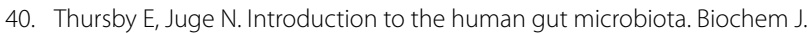
2017:474(11):1823-36.

41. Thomas MSC, Denis M, Iroise D. Educational neuroscience: development across the life span. London: Routledge; 2020.

42. American Psychiatric Association. Diagnostic and statistical manual of mental disorders (DSM-5 $\left.{ }^{\circledR}\right)$. Washington: American Psychiatric Pub; 2013.

43. Lan G, Benito-Picazo J, Roijers DM, Dominguez E, Eiben AE. Real-time robot vision on low-performance computing hardware. In 2018 15th International Conference on Control, Automation, Robotics and Vision (ICARCV) 2018 Nov 18 (pp. 1959-1965). IEEE

44. Li X, Haoyang Liu X, Zhao GZ, Xing C. Automatic approach for constructing a knowledge graph of knee osteoarthritis in Chinese. Health Inform Sci Syst. 2020;8(1):1-8.

45. Lan G, De Vries L, Wang S. Evolving efficient deep neural networks for real-time object recognition. In 2019 IEEE Symposium Series on Computational Intelligence (SSCI), pp. 2571-2578; 2019

46. Lan G, De Carlo M, van Diggelen F, Tomczak JM, Roijers DM, Eiben AE. Learning directed locomotion in modular robots with evolvable morphologies. arXiv:2001.07804. 2020.

47. Koivu A, Sairanen M. Predicting risk of stillbirth and preterm pregnancies with machine learning. Health Inform Sci Syst. 2020;8(1):1-12.

48. Lan G, Chen J, Eiben AE. Simulated and real-world evolution of predator robots. In 2019 IEEE Symposium Series on Computational Intelligence (SSCI), 2019; pp. 1974-1981.

49. Lan G, Chen J, Eiben AE. Evolutionary predator-prey robot systems: From simulation to real world. In Proceedings of the Genetic and Evolutionary Computation Conference Companion, 2019; pp. 123-124.

50. Lan G, Tomczak JM, Roijers DM, Eiben AE. Time efficiency in optimization with a bayesian-evolutionary algorithm. arXiv:2005.04166. 2020

Publisher's Note Springer Nature remains neutral with regard to jurisdictional claims in published maps and institutional affiliations. 